Marquette University

\title{
e-Publications@Marquette
}

School of Dentistry Faculty Research and

Publications

Dentistry, School of

$1-15-2019$

\section{Mathematical Modeling of Oxygen Transfer in Porous Scaffolds for Stem Cell Growth: The Effects of Porosity, Cell Type, Scaffold Architecture and Cell Distribution}

\author{
Saeid Atashrouz \\ Amirkabir University of Technology-Iran \\ Ali Hatampoor \\ Amirkabir University of Technology-Iran \\ Amir Yadegari \\ Marquette University \\ Hamed Ghasemi \\ Tehran University of Medical Sciences \\ Lobat Tayebi \\ Marquette University, lobat.tayebi@marquette.edu
}

See next page for additional authors

Follow this and additional works at: https://epublications.marquette.edu/dentistry_fac

Part of the Dentistry Commons

\section{Recommended Citation}

Atashrouz, Saeid; Hatampoor, Ali; Yadegari, Amir; Ghasemi, Hamed; Tayebi, Lobat; and Rasoulianboroujeni, Morteza, "Mathematical Modeling of Oxygen Transfer in Porous Scaffolds for Stem Cell Growth: The Effects of Porosity, Cell Type, Scaffold Architecture and Cell Distribution" (2019). School of Dentistry Faculty Research and Publications. 375.

https://epublications.marquette.edu/dentistry_fac/375 


\section{Authors}

Saeid Atashrouz, Ali Hatampoor, Amir Yadegari, Hamed Ghasemi, Lobat Tayebi, and Morteza

Rasoulianboroujeni

This article is available at e-Publications@Marquette: https://epublications.marquette.edu/dentistry_fac/375 
Marquette University

e-Publications@Marquette

\section{Dentistry Faculty Research and Publications/School of Dentistry}

This paper is NOT THE PUBLISHED VERSION; but the author's final, peer-reviewed manuscript. The published version may be accessed by following the link in the citation below.

Materials Chemistry and Physics, Vol. 222 (January 15, 2019): 377-383. DOI. This article is @ Elsevier and permission has been granted for this version to appear in e-Publications@Marquette. Elsevier does not grant permission for this article to be further copied/distributed or hosted elsewhere without express permission from Elsevier.

\section{Mathematical Modeling of Oxygen Transfer in Porous Scaffolds for Stem Cell Growth: The Effects of Porosity, Cell Type, Scaffold Architecture and Cell Distribution}

\section{Saeid Atashrouz}

Department of Chemical Engineering, Amirkabir University of Technology, Tehran, Iran

Ali Hatampoor

Department of Biomedical Engineering, Amirkabir University of Technology, Tehran, Iran

Amir Yadegari

Marquette University School of Dentistry, Milwaukee, WI

Hamed Ghasemi

Eye Research Center, Farabi Eye Hospital, Tehran University of Medical Sciences, Tehran, Iran Lobat Tayebi

Marquette University School of Dentistry, Milwaukee, WI

Morteza Rasoulianboroujeni

Department of Biomedical Engineering, Amirkabir University of Technology, Tehran, Iran 


\section{Abstract}

Oxygen plays a key role in human mesenchymal stem cell growth. Without adequate oxygen (hypoxic condition), cells are not able to survive, proliferate, and migrate. The objective of the present study is to investigate oxygen transfer through the cell-seeded scaffolds stored in static or dynamic bioreactors using a mathematical model. The effects of porosity, cell type, scaffold architecture and cell distribution as potential effective parameters on oxygen transfer kinetics were examined. The results suggest the substantial effect of porosity and cell type on the oxygen concentration within the scaffold compared to scaffold architecture (homogeneous vs. gradient). The obtained data show that the direction of oxygen transfer in deep regions with dead cells changes over time and reverse mass transfer allows the cells to nourish from both top and bottom layers. Finally, the extent of oxygen transfer in static bioreactors/cultures was compared to dynamic ones. The results show that dynamic bioreactors have a better performance and are more efficient for oxygen transfer.

\section{Graphical abstract}

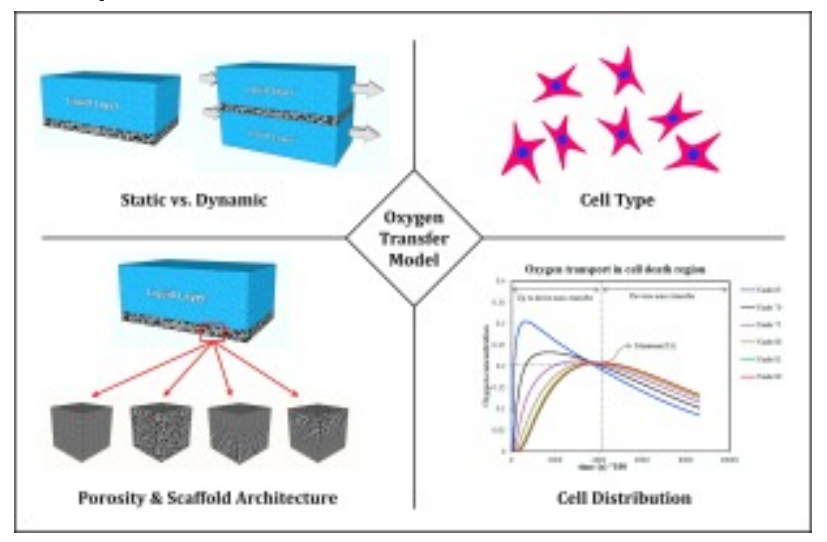

\section{Keywords}

Oxygen transfer, Gradient scaffold, Oxygen consumption, Porosity, Mathematical modeling

\section{Introduction}

For many years, patients have confronted with tissue or organ loss due to various diseases [1]. It has been reported that more than one million patients are annually suffering from tissue loss in United States [2] and seventeen people die every day due to lack of available organ donors [3].

Despite the lifesaving performance of autografts and allografts in some cases, they are mostly limited by the number of available tissues/organs for transplantation and rejection of transplant by patient's immune system [4]. Tissue engineered constructs as a potential alternative have recently attracted considerable attention. Porous scaffolds are known as one of the most important elements in tissue engineering as they grant necessary area for cell adhesion, growth, and proliferation [5] as well as mechanical support for the whole construct.

Diffusion of oxygen is crucial for enhancing the performance of scaffolds by improving cell adhesion and proliferation [6]. It goes without saying that inadequate oxygen concentration in certain areas of a 
porous scaffold may lead to cessation of cell proliferation and triggering cell death [7]. Generally, in hypoxic condition, cells prone to convert glucose to lactic acid which leads to necrosis [8]. It has been reported that some cells can tolerate the mild hypoxic condition for several hours; however, they cannot survive in zero oxygen concentration more than a few minutes [5]. Thus, examination and optimization of oxygen concentration in porous scaffolds play a pivotal role in improving the performance of them. As a common strategy, porous scaffolds are fabricated in small sizes to facilitate the diffusion of oxygen throughout the construct [9]. However, for critical size scaffolds, the uniform distribution of oxygen is not always possible. The oxygen concentration gradient in such scaffolds is a function of exposure time and distance from the oxygen source $[5,10]$. The location of the oxygen source and the efficiency of oxygen transport can influence the uniformity of cell distribution all over the scaffold by formation of hyper or hypoxic condition in certain regions [5].

Scaffolds fabricated through conventional methods typically possess uniform porosity and pore size all over the construct [9]. In contrast, anisotropic scaffolds including scaffolds with pore size and/or porosity gradient have received wide attention in recent years [[11], [12], [13], [14]]. Such scaffolds have been reported to significantly increase the seeding efficiency and prompt a more homogenous cell distribution compared to homogeneous ones $[11,15]$. Such observations can be explained by the differences in scaffold architecture which in turn influences flow conditions as well as oxygen and nutrient transfer throughout the scaffold [16]. Modeling of oxygen (or nutrients) concentration in gradient scaffolds vs. corresponding homogenous ones can lead to better comprehension of the effect of scaffold architecture on the cell growth, proliferation and distribution through governing the mass transfer throughout the scaffold [11,17]. There are a few number of reports on the modeling of oxygen concentration in various scaffolds [2,[18], [19], [20]]. However, no model has been developed for predicting oxygen diffusion in gradient scaffolds. In the present study, in addition to investigating the effect of porosity, cell type and distribution, a mathematical model was developed to predict the oxygen concentration through homogenous and gradient scaffolds. Spatial and time dependencies of oxygen concentration have also been investigated. Finally, the effect of static and dynamic bioreactors on oxygen transfer and concentration profile was mathematically studied.

\section{Methods/mathematical modeling}

The bioreactor with similar equations of mass transfer for a constant porosity was employed as previously reported by Zhao et al. [17]. The mass transfer equations were also modified to obtain relevant mathematical models for the systems via varying porosity/pore size such as gradient scaffolds. The systems have been modeled both in static and dynamic conditions as schematically represented in Fig. 1. 

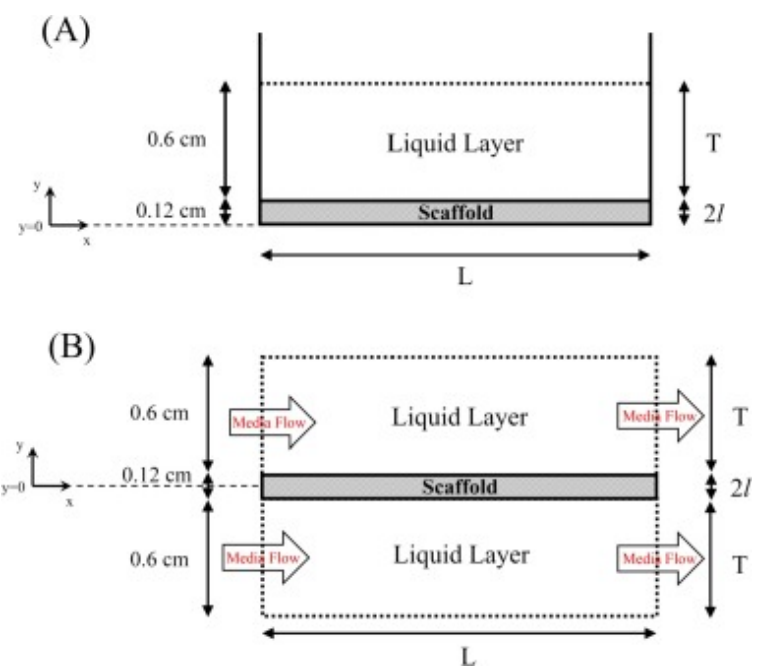

Fig. 1. Schematic illustration of proposed systems for static (A) and dynamic (B) bioreactors [17] (with permission from Wiley).

\subsection{Static bioreactor/culture}

According to Fig. 1(A), the bioreactor is divided into two separate regions: liquid layer and scaffold-cell layer. Due to absence of cells in liquid layer, there is no reaction in this region. Consequently, the mass transfer equation is defined as follows:

$$
\frac{\partial c}{\partial t}=\left(\nabla D_{L} \nabla c\right)
$$

where $c$ is dimensionless oxygen concentration which is obtained through dividing the oxygen concentration by the dissolved oxygen concentration in equilibrium with atmospheric oxygen, $t$ is time, and $\mathrm{DL}$ is oxygen diffusion coefficient in liquid layer.

For scaffold-cell layer, an extra reaction term is added to equation (1) due to the consumption of oxygen by the cells:

(2)

$$
\frac{\partial c}{\partial t}=\left(\nabla D_{S} \nabla c\right)-\frac{Q_{m} K_{e q} \varepsilon_{\gamma} c}{\frac{K_{m}}{C_{0}}+K_{e q} c \varepsilon_{\gamma}}
$$

where $D_{S}$ is diffusion coefficient of oxygen in scaffold. The reaction term is attributed to the consumption of oxygen and assumed to follow Michaelis-Menten kinetics where $Q_{m}$ and $K_{m}$ are specific oxygen consumption rate and saturation constant, respectively. $K_{e q}$ is equilibrium constant for oxygen and $C_{0}$ is dissolved oxygen concentration in equilibrium with atmospheric oxygen. The volume fraction of cells is represented by $\varepsilon_{\gamma}$ which can be obtained through following equation: 
$\varepsilon_{\gamma}=\varepsilon_{\gamma_{0}} \exp (m t)$

where $\varepsilon_{\gamma_{0}}$ is initial cell volume fraction and $\mathrm{m}$ is cell growth rate.

The boundary conditions in equations (1), (2) are defined as follows: Oxygen concentration in the top edge of liquid layer is equal to oxygen concentration in incubator (Catm):

(4)

$c=C_{a t m} \forall x=[0, L], \mathrm{y}=2 \mathrm{I}+\mathrm{T}$

Also, an equal diffusion flux between liquid layer and scaffold-cell layer is considered at the interface of layers:

(5)

$-\left.D_{\beta} \nabla c\right|_{\text {liquidlayer }}=-\left.D_{e f f} \nabla c\right|_{\text {celllayer }}$

Finally, there is no mass transfer to solid walls:

$\nabla D_{e f f} c=0 \forall y=[0,2 l], \mathrm{x}=0$

(7)

$\nabla D_{e f f} c=0 \forall y=[0,2 l], \mathrm{x}=\mathrm{L}$

$\nabla D_{e f f} c=0 \forall x=[0, L], y=0$

\subsection{Dynamic bioreactor}

The dynamic bioreactor is schematically illustrated in Fig. 1(B). A membrane is inserted at the top and bottom of the bioreactor for oxygen exchange. In the liquid layer, oxygen transfer is accomplished through both diffusion and convection mass transfer. To model the whole system, only half of the bioreactor is taken into account due to symmetry of the bioreactor. The mass transfer equation in the liquid layer is defined as:

$$
\frac{\partial c}{\partial t}=\left(\nabla D_{L} \nabla c\right)-v_{x} \nabla c
$$

where $v_{x}$ is the velocity of media in liquid layer which is defined using the following equation: 
$v_{x}=\frac{6 v_{\text {ave }}}{T^{2}}\left[(T+2 l) y-y^{2}-\left(l^{2}+T l\right)\right]$

In this equation $v_{\text {ave }}$ stands for the average velocity of the media.

In contrast to the liquid layer, in the scaffold-cell layer, oxygen is only transferred through diffusion mechanism alike the static bioreactor/culture:

$\frac{\partial c}{\partial t}=\left(\nabla D_{S} \nabla c\right)-\frac{Q_{m} K_{e q} \varepsilon_{\gamma} c}{\frac{K_{m}}{C_{0}}+K_{e q} c \varepsilon_{\gamma}}$

Oxygen flux on top of the liquid layer can be written as a function of membrane permeability as follows [17]:

$-D_{\beta} \frac{\partial c}{\partial y}=\operatorname{Perm}\left(C_{a t m}-c\right)$

The flux balance equation at the inlet of reactor is considered according to Danckwert's boundary condition [21]:

$v c_{i n}=v c-D_{L} \frac{\partial c}{\partial x} \forall y=[l, l+T], \mathrm{x}=0$

where $c_{i n}$ is the concentration of dissolved oxygen delivered at the inlet which is equal to $C_{\text {atm. }}$.

It should be noted that, a zero-diffusive mass transfer is considered at the end of reactor:

$D_{L} \frac{\partial c}{\partial x}=0 \forall y=[l, l+T], \mathrm{x}=\mathrm{L}$

Like static condition, an equal diffusion flux between liquid layer and cell layer is considered the interface of the layers. Furthermore, mass transfer of oxygen to solid walls is equal to zero:

$\nabla D_{e f f} c=0 \forall y=[0, l], \mathrm{x}=0$

$\nabla D_{e f f} c=0 \forall y=[0, l], \mathrm{x}=\mathrm{L}$ 


$$
\nabla D_{e f f} c=0 \forall x=[0, L], y=0
$$

\subsection{Effect of porosity and scaffold architecture on mass transfer}

In the current study, the effect of porosity was examined by modifying the aforementioned mass transfer equations. Equations such as equation (2) are not capable of reflecting the effect of porosity, which makes them unfit for modeling of mass transfer in porous constructs. The effect of porosity was investigated by employing an oxygen transfer model for brain tissue developed by Nicholson [22]:

$\frac{\partial c}{\partial t}=D^{*} \nabla^{2} c+\frac{R}{\alpha}$

where $D^{*}=D / \lambda^{2}$ is the effective diffusion coefficient and $\lambda$ represents tortuosity of the porous construct. In this equation, $R$ and $\alpha$ symbolize reaction term and porosity of the system, respectively. Based on equation (18), the changes in oxygen concentration in a porous media can be predicted.

\subsection{Solution procedure}

The explicit finite difference method was used to solve the mass transfer equation in this study. Indeed, mass transfer equation and the related boundary conditions were developed and calculated by using MATLAB programming software. In the present study, human mesenchymal stem cells were considered as the seeded cells into the scaffolds. Characteristics of static and dynamic systems are summarized in Table 1. Generally, for solving an equation using analytical methods, time is a negligible parameter. However, time plays an important role for solving the mathematical equations using numerical methods. Herein, we presented two different networks including $30 \times 30$ and $100 \times$ 100 nodes in width and length of bioreactor for solving the mass transfer equations. It should be mentioned that the number of nodes significantly affects the required time for solving the equations. The problem was separately solved for each network in static bioreactor/culture and the results are shown in Fig. 2. While $100 \times 100$ node network needs more time for calculations, the final results revealed no significant difference between $30 \times 30$ and $100 \times 100$ node networks suggesting that using smaller networks would reduce the time of calculation. We even tried higher number of nodes and no difference in the outcome was observed (data not shown). Therefore, $30 \times 30$ node network was selected for discretizing of the system and predicting oxygen mass transfer in porous scaffolds in the present study.

Table 1. Characteristics of static and dynamic bioreactors.

\begin{tabular}{|l|l|}
\hline Parameter & Value \\
\hline Static Bioreactor & \\
\hline Specific cell volume & $V_{g}=2.8 \times 10^{-9}$ \\
\hline Cell growth rate & $m=3.87454 \times 10^{-7} 1 / \mathrm{s}$ \\
\hline Initial cell volume fraction & $\varepsilon_{\gamma o}=9.65 \times 10^{-3}$ \\
\hline $\begin{array}{l}\text { Oxygen concentration in equilibrium with } 20 \% \\
\text { gas-phase oxygen }\end{array}$ & $C_{0}=2.10 \times 10^{-7} \mathrm{mmol} / \mathrm{cm}^{3}$ \\
\hline
\end{tabular}




\begin{tabular}{|l|l|}
\hline Oxygen diffusion coefficient in liquid layer & $D_{\beta}=3.29 \times 10^{-5} \mathrm{~cm}^{2} / \mathrm{s}$ \\
\hline $\begin{array}{l}\text { Oxygen diffusion coefficient through cells in } \\
\text { the scaffold }\end{array}$ & $D_{\gamma}=1.59 \times 10^{-5} \mathrm{~cm}^{2} / \mathrm{s}$ \\
\hline Scaffold thickness & $2 l=0.12 \mathrm{~cm}$ \\
\hline Liquid layer thickness & $T=0.6 \mathrm{~cm}$ \\
\hline Bioreactor length & $L=10 \mathrm{~cm}$ \\
\hline Bioreactor width & $w=2.5 \mathrm{~cm}$ \\
\hline Saturation constant & $K_{m}=1.105265 \times 10^{-8} \mathrm{~mol} / \mathrm{cm}^{3}$ \\
\hline Dynamic Bioreactor & \\
\hline Specific cell volume & $V_{g}=2.8 \times 10^{-9}$ \\
\hline Saturation constant & $K_{m}=1.105265 \times 10^{-8} \mathrm{~mol} / \mathrm{cm}^{3}\left(=0.05 C_{0}\right)$ \\
\hline Cell growth rate & $m=1.18 \times 10^{-6} 1 / \mathrm{s}$ \\
\hline Initial cell volume fraction & $\varepsilon_{\gamma o}=1.53 \times 10^{-3}$ \\
\hline $\begin{array}{l}\text { Dimensionless specific oxygen consumption } \\
\text { rate }\end{array}$ & $Q_{m}(1 / \mathrm{s})=-3.6198 \times 10^{-16} t^{2}$ \\
\hline Matrix scaffold & \\
\hline Liquid layer thickness & $2 l=0.12 \mathrm{~cm}$ \\
\hline Bioreactor length & $T=0.6 \mathrm{~cm}$ \\
\hline Bioreactor width & $L=10 \mathrm{~cm}$ \\
\hline Flow rate & $w=2.5 \mathrm{~cm}$ \\
\hline Membrane thickness & $0.1 \mathrm{ml} / \mathrm{min}$ \\
\hline Membrane length & $130 \mu \mathrm{m}$ \\
\hline Membrane permeability & $10 \mathrm{~cm}$ \\
\hline Average velocity & $P e r m=1.343 \times 10^{-3}$ \\
\hline & $1.11 \times 10^{-3} \mathrm{~cm} / \mathrm{s}$ \\
\hline & \\
\hline &
\end{tabular}

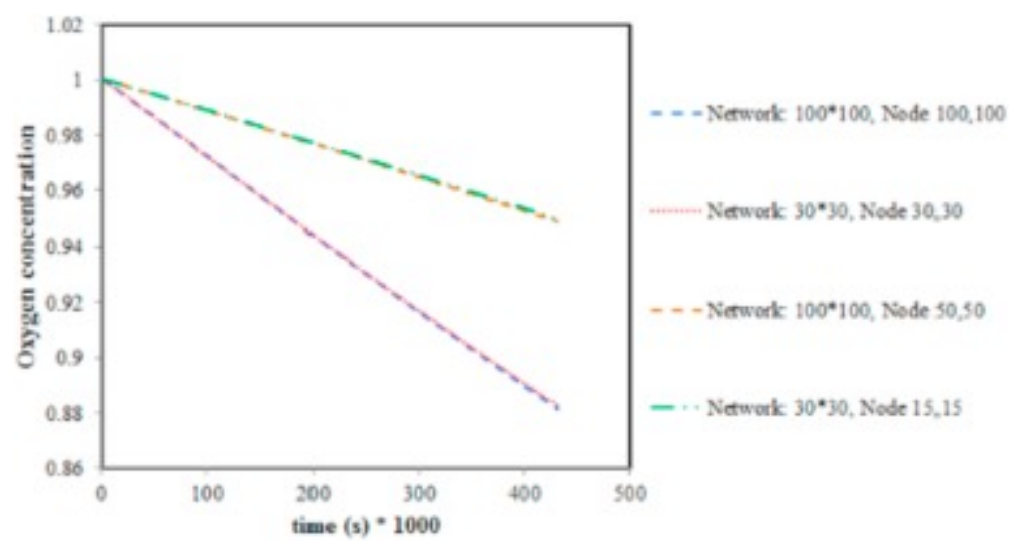

Fig. 2. Effect of number of nodes on the outcome of the model.

\section{Results and discussion}

In the present study, we have focused more on the results of oxygen mass transfer in static bioreactor/culture. However, we compared the performance of both static and dynamic bioreactors at the end. Fig. 3(A) shows the oxygen profile after 40 days in static condition when the effect of porosity is neglected. As expected, the minimum value of oxygen concentration belongs to the lowest layer of scaffold. It should be noted that in the liquid layer, the oxygen concentration is approximately a linear 
function of liquid depth. Nevertheless, the non-linear behavior in scaffold region can be attributed to oxygen consumption (equation (2)). Also, there is no oxygen diffusion in the length of bioreactor due to the dominant boundary conditions in the system. Fig. 3(A) gives the time required for complete oxygen consumption in the last node i.e. the last layer of scaffold. As seen, the oxygen concentration drops down to zero approximately after $5 \times 106$ seconds ( $~ 58$ day). The variation of oxygen concentration as a function of depth at different time intervals has been presented in Fig. 3(B).

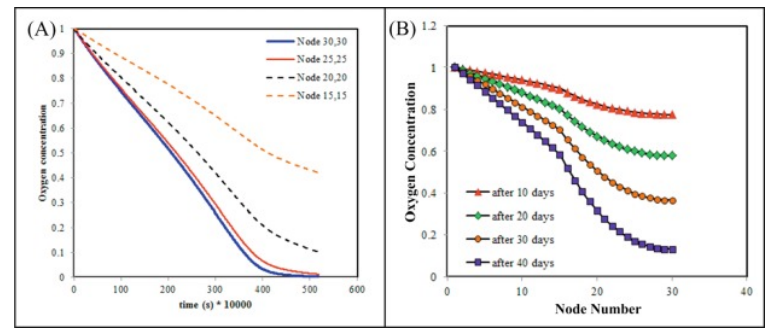

Fig. 3. Oxygen concentration as a function of time in different depths of a scaffold to find the time for complete oxygen consumption in the last layer of the scaffold $(A)$; oxygen concentration as a function of depth of the system at various time points (B).

$\left(Q_{m}=0.0001\right.$, the effect of porosity has been neglected $)$.

Estimation of the required time to reach a certain concentration at different depths of a scaffold is of great importance since it facilitates detection of hypoxic condition in each layer leading to initiation of cell death. It can be obviously seen in Fig. 3(B) that the difference between oxygen concentration in the upper and lower regions of the system increases over time owing to the oxygen transfer and consumption in the scaffold. Such an observation also suggests a higher rate of oxygen consumption compared to oxygen diffusion.

\subsection{Effect of porosity and cell type}

In the next step, the effect of porosity on oxygen concentration was investigated. The general mass transfer equation in this case was described as equation (18). Fig. 4 shows the effect of $Q_{m}$ and porosity on oxygen concentration in the last layer of scaffold after one day. Specific oxygen consumption rate $\left(Q_{m}\right)$ plays an important role in the calculations and depends on type of the cells. As seen in Fig. 4, the oxygen concentration is very sensitive to $Q_{m}$ and an increase in $Q_{m}$ results in significant decrease in oxygen concentration. On the other hand, oxygen concentration increases by increasing the porosity of scaffolds. Equation (18) can be rewritten in the following form to facilitate the analysis of the system:

$\alpha \frac{\partial C}{\partial t}=\alpha D^{*} \nabla^{2} C+R$ 

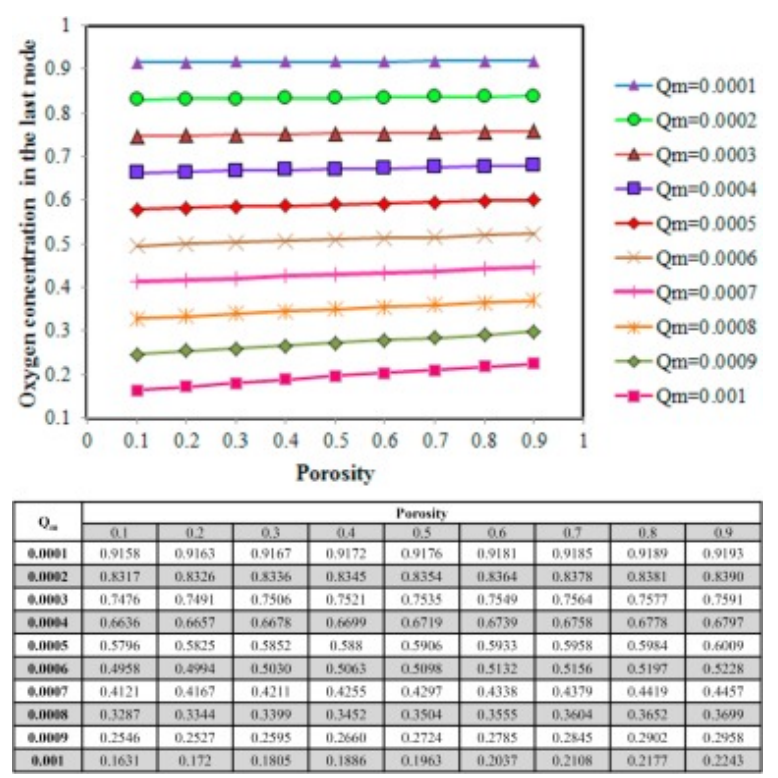

Fig. 4. Variation of oxygen concentration with different porosities and specific oxygen consumption rate $\left(Q_{m}\right)$ for the last node after one day (the calculated oxygen concentrations have been included in the table).

The term $\alpha D^{*}$ is effective diffusion coefficient $\left(D_{e f f}\right)$. The scaffold with higher porosity possesses higher effective diffusion coefficient which means more oxygen diffusion into the system. By assuming equal oxygen consumption rate $(R)$ for two systems with different porosities, oxygen concentration versus porosity shows an ascending behavior as illustrated in Fig. 4 . It should be noted that by increasing the value of $Q_{m}$, the effect of porosity on oxygen concentration increases while for small values of $Q_{m}$ (such as 0.0001 ), porosity approximately has no significant effect on the oxygen concentration. It should be mentioned that the effect of porosity on oxygen concentration presented in Fig. 4 was examined by assuming a constant porosity for the whole scaffold.

\subsection{Effect of gradient architecture}

In the next step, gradient scaffolds were taken into the account. Unlike homogenous scaffolds, in gradient ones, the porosity of scaffold in different regions is not constant. The effect of scaffold architecture on oxygen concentration was investigated for two types of gradient (Grad 1 and 2) and two types of homogenous (Homog 1 and 2) scaffolds as schematically illustrated in Fig. 5 . The porosities of homogenous scaffolds 1 and 2 were assumed to be 0.9 and 0.1 , respectively. The porosity of gradient scaffolds was supposed to change from 0.1 to 0.9 with the increment value of 0.1 . The values of oxygen concentration after 3 days at different nodes of the scaffolds are presented in Fig. 5. It should be noted that more than 30 nodes are required to examine the effect of porosity change on the oxygen concentration profile. Hence, a 90-node network was used in which the nodes from 45 to 90 represent the scaffold region. As seen in Fig. 5, oxygen concentration within both Grad 1 and Grad 2 lies between Homog 1 and Homog 2 scaffolds. Though not significantly, Grad 2 had a slightly better performance in oxygen delivery to the underneath layers than Grad 1. It should be noted that the reported data has been obtained based on the given parameters used in this study. Changing any of these parameters may or may not significantly affect the outcome. Furthermore, oxygen concentration is not the only factor that influences scaffold performance. As an example, while scaffolds with very big pore size allow facilitated diffusion of oxygen and nutrient into the underneath layers, they may 
provide inadequate surface area for cell adhesion. Taking this fact in to the account, oxygen concentration profile might be one of the origins of the observed difference in biological performance among various homogenous or gradient scaffold [11], but not necessarily the main origin.

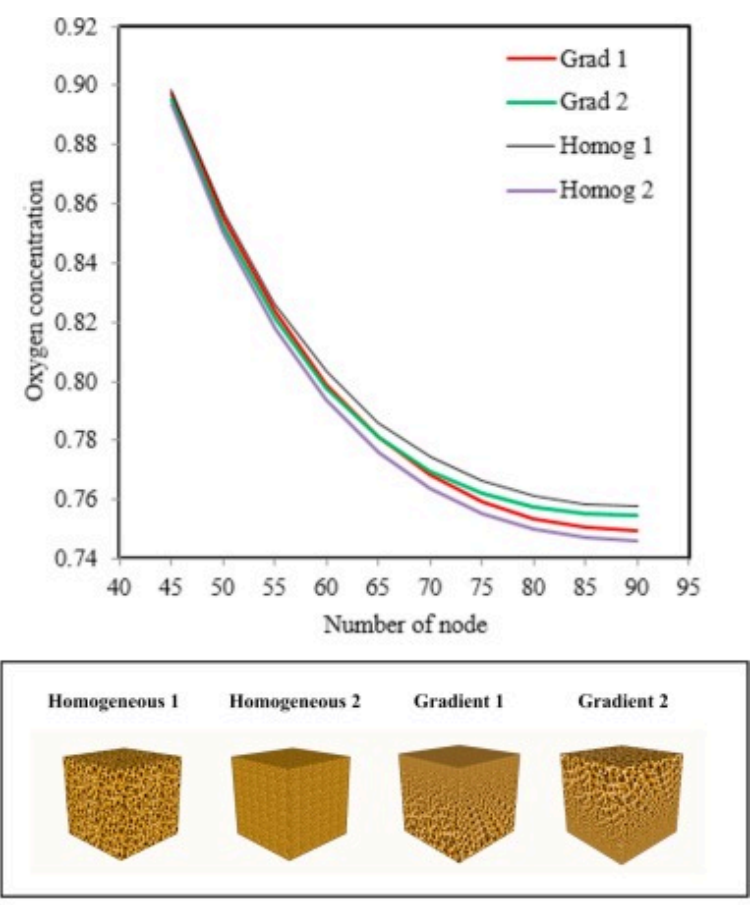

\begin{tabular}{|c|c|c|c|c|}
\hline Node Number & Homog 1 & Homog 2 & Grad 1 & Grad 2 \\
\hline $\mathbf{4 5}$ & 0.8938 & 0.8985 & 0.8973 & 0.8951 \\
\hline $\mathbf{5 0}$ & 0.8508 & 0.8574 & 0.8555 & 0.8528 \\
\hline $\mathbf{5 5}$ & 0.8180 & 0.8262 & 0.8234 & 0.8210 \\
\hline $\mathbf{6 0}$ & 0.7937 & 0.8030 & 0.7991 & 0.7977 \\
\hline $\mathbf{6 5}$ & 0.7760 & 0.7861 & 0.7812 & 0.7811 \\
\hline $\mathbf{7 0}$ & 0.7636 & 0.7743 & 0.7684 & 0.7697 \\
\hline $\mathbf{7 5}$ & 0.7552 & 0.7663 & 0.7594 & 0.7623 \\
\hline $\mathbf{8 0}$ & 0.7499 & 0.7613 & 0.7536 & 0.7578 \\
\hline $\mathbf{7 5}$ & 0.7470 & 0.7586 & 0.7503 & 0.7554 \\
\hline $\mathbf{9 0}$ & 0.7462 & 0.7578 & 0.7494 & 0.7548 \\
\hline
\end{tabular}

Fig. 5. Oxygen concentration for homogeneous and gradient scaffolds after 3 days along with schematic illustration of considered scaffolds (the calculated values of oxygen concentration have been included in the table).

\subsection{Penetration depth and cell distribution}

Cell penetration/migration/distribution all over the scaffold is also of great importance in tissue engineering. After seeding the cells onto the scaffold, they start to grow and migrate depending on the presented stimuli/signals in the surrounding environment. Whether the cells migrate instinctively to a certain depth within the scaffold (or even the last layer) or they are artificially distributed all over the construct by means of seeding techniques, they need oxygen to be present in their surrounding media for survival. To investigate the effect of penetration depth and cell distribution, two different conditions were considered:

1 Cells cover the entire scaffold. When the hypoxic condition is reached, the cells in deeper layers of the scaffold die and only cells within the top $1 / 3^{\text {rd }}$ of scaffold thickness can survive. In this case, the oxygen concentration in regions where there is no cell is 0 . 
2 Cells only cover the top $1 / 3^{\text {rd }}$ of scaffold thickness. It is assumed that cell death does not occur in this condition. Initial oxygen concentration in regions of scaffold with no cell is equal to 1.

It should be noted that initial oxygen concentration in other regions of the scaffold and liquid layer for both conditions was assumed to be 1 .

Fig. 6(A and $B)$ displays oxygen concentration for the first condition after ten days. Axes $X, Y$ and $Z$ represent depth, length of bioreactor, and oxygen concentration, respectively. Since measuring oxygen concentration in scaffolds is a complicated procedure, experimental data is not available for comparing the predicted values in this study with real experimental data. However, the trend of the obtained values is similar to the previous study by Zhao et al. [17]. Fig. 6(C) shows oxygen concentration as a function of time for different nodes of static bioreactor for the first condition. In nodes 1-60 attributed to the liquid layer and living cells, oxygen concentration decreases over time while at deeper nodes indicating regions with no active cells, it is contrary. Fig. 6(D) shows the oxygen concentration in region without cells for the first condition. According to the figure, oxygen concentration increases to reach a plateau before decreasing. This maximum value is different for various nodes and decreases at deeper parts of the scaffold. Therefore, the smallest maximum value is for node number 90 which is the deepest part of scaffold. Before the maximum value of oxygen concentration is reached, oxygen mass transfer is from the top of the bioreactor to the bottom. As the oxygen concentration reaches its maximum value, the direction of mass transfer reverses providing oxygen for cells from both top and bottom. The reverse mass transfer can improve oxygen transfer to the last layer of the cells residing in the top $1 / 3^{\text {rd }}$ of the scaffold.
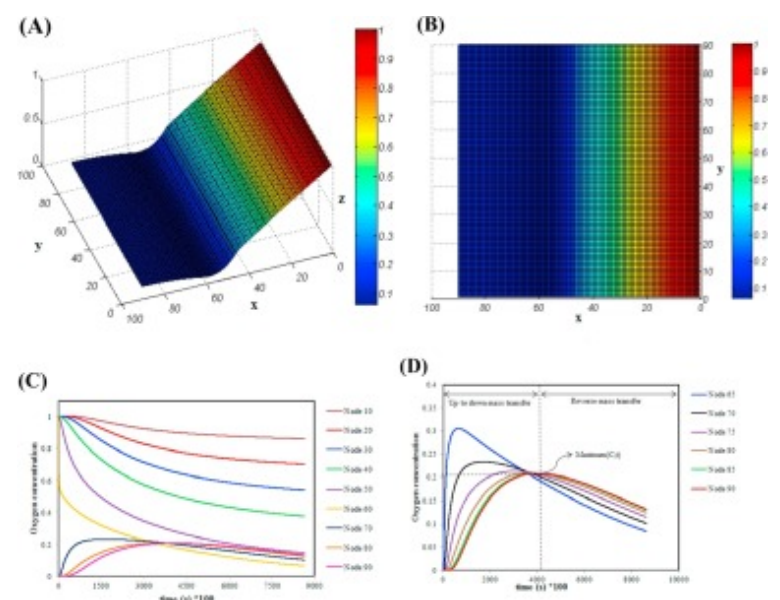

(D)

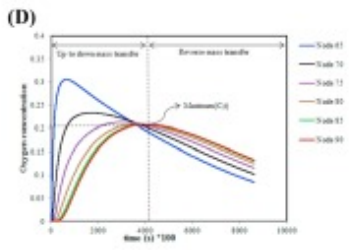

Fig. 6. 3D (A) and 2D (B) representation of oxygen concentration for first condition after 10 days. Variation of oxygen concentration over time for the first condition in the whole system (C) and the region where dead cells are located (D). Cells cover the entire scaffold but only cells within the top $1 / 3^{\text {rd }}$ of scaffold thickness can survive. The oxygen concentration in regions where there is no cell is 0 .

Fig. 7(A and B) shows the oxygen concentration for the second condition. As presented in the figure, oxygen concentration in the last layer is higher than the previous condition (Fig. 6) due to the initial oxygen concentration in this region. Fig. 7(C) shows oxygen concentration for regions with no cell. The extremum point observed in previous condition does not appear for the second condition and there is no reverse mass transfer. 

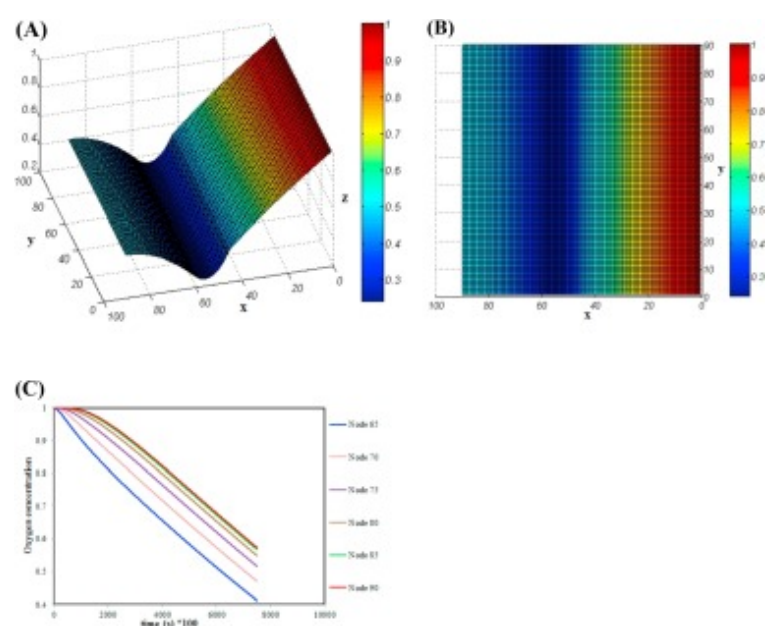

Fig. 7. 3D (A) and 2D (B) representation of oxygen concentration for second condition after 10 days. Variation of oxygen concentration over time for the second condition in regions with no cell (C). Cells only cover the top $1 / 3$ rd of scaffold thickness. Initial oxygen concentration in regions of scaffold with no cell is equal to 1 .

\subsection{Static vs. dynamic bioreactor/culture}

Fig. 8 reveals the comparison between the performance of static versus dynamic bioreactors. This figure shows the variations of oxygen concentration in the last layer of static and dynamic bioreactors as a function of time. As seen, the oxygen concentration in a static bioreactor decreases with a significantly higher slope compared to a dynamic bioreactor suggesting that dynamic bioreactors present a better performance for oxygen transfer. It has been recently reported that cell volume fraction achieved when dynamic reactors are used, is much higher than that from static reactor due to better diffusion and distribution of oxygen molecules throughout the reactor [17].

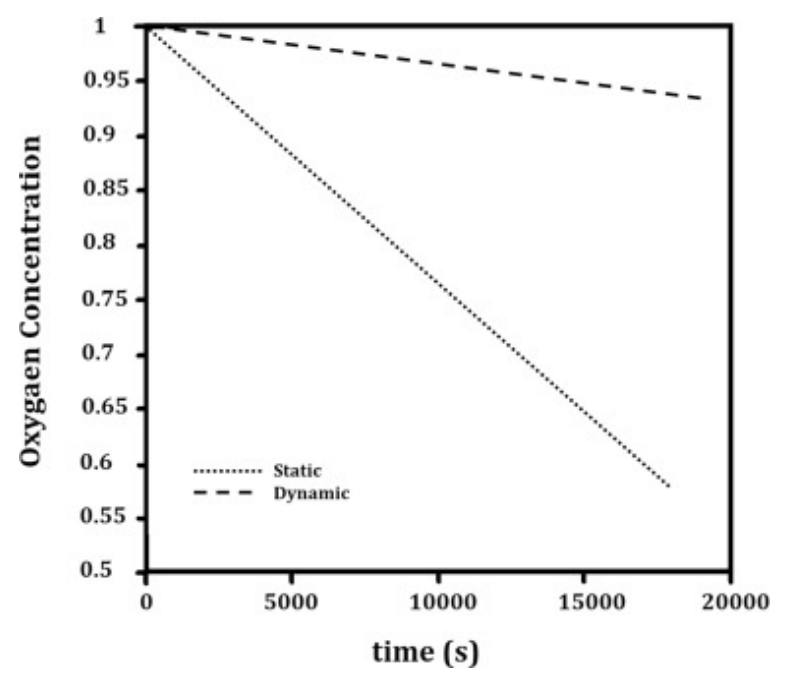

Fig. 8. Comparison of variation of oxygen concentration in the last layer in static and dynamic bioreactors over time.

\section{Conclusion}

In the present study, oxygen transfer kinetics and the effective parameters on the oxygen concentration within human mesenchymal stem cell seeded tissue engineering scaffolds were mathematically studied. A mass transfer equation was proposed for evaluating the effect of porosity in 
modeling oxygen transfer in bioreactor. The model can predict the oxygen concentration as a function of time in different regions of a bioreactor. Such information can help recognize the important factors as well as the scope of their effect on mass transfer within a scaffold which in turn influences cell viability and function including proliferation and migration. The results showed that porosity plays an important role in oxygen transfer especially when specific oxygen consumption rate $\left(Q_{m}\right)$ has a big value for the cell of interest. Furthermore, the results indicated that by occurring cell death in deep layers of the scaffold, oxygen transfer direction changes over time and reverse mass transfer occurs. A comparison between static and dynamic bioreactors suggested that the dynamic bioreactors are more efficient compared to static bioreactors in terms of oxygen transfer.

\section{Acknowledgement}

The authors declare no conflict of interest.

\section{References}

[1] S. Cai, J. Xi. A control approach for pore size distribution in the bone scaffold based on the hexahedral mesh refinement. Comput. Aided Des., 40 (2008), pp. 1040-1050

[2] C.J. Galban, B.R. Locke. Effects of spatial variation of cells and nutrient and product concentrations coupled with product inhibition on cell growth in a polymer scaffold. Biotechnol. Bioeng., 64 (1999), pp. 633-643

[3] B.J. Lawrence. Mass Transfer in Porous Tissue Engineering Scaffolds. Oklahoma State University (2008)

[4] C.M. Murphy, F.J. O'Brien. Understanding the effect of mean pore size on cell activity in collagenglycosaminoglycan scaffolds. Cell Adhes. Migrat., 4 (2010), pp. 377-381

[5] K.A. Landman, A.Q. Cai. Cell proliferation and oxygen diffusion in a vascularising scaffold. Bull. Math. Biol., 69 (2007), pp. 2405-2428

[6] A. Hadjizadeh, C.J. Doillon. Directional migration of endothelial cells towards angiogenesis using polymer fibres in a 3D co-culture system. J Tissue Eng Regen Med, 4 (2010), pp. 524-531

[7] K. Kubo, N. Tsukimura, F. Iwasa, T. Ueno, et al. Cellular behavior on TiO 2 nanonodular structures in a micro-to-nanoscale hierarchy model. Biomaterials, 30 (2009), pp. 5319-5329

[8] M. Pisu, N. Lai, A. Cincotti, A. Concas, et al. Modeling of engineered cartilage growth in rotating bioreactors. Chem. Eng. Sci., 59 (2004), pp. 5035-5040

[9] T. Woodfield, C.V. Blitterswijk, J.D. Wijn, T. Sims, et al. Polymer scaffolds fabricated with pore-size gradients as a model for studying the zonal organization within tissue-engineered cartilage constructs. Tissue Eng., 11 (2005), pp. 1297-1311

[10] P. Pathi, T. Ma, B.R. Locke. Role of nutrient supply on cell growth in bioreactor design for tissue engineering of hematopoietic cells. Biotechnol. Bioeng., 89 (2005), pp. 743-758

[11] J.M. Sobral, S.G. Caridade, R.A. Sousa, J.F. Mano, et al. Three-dimensional plotted scaffolds with controlled pore size gradients: effect of scaffold geometry on mechanical performance and cell seeding efficiency. Acta Biomater., 7 (2011), pp. 1009-1018

[12] B.A. Harley, A.Z. Hastings, I.V. Yannas, A. Sannino. Fabricating tubular scaffolds with a radial pore size gradient by a spinning technique. Biomaterials, 27 (2006), pp. 866-874

[13] M. Silva, L. Cyster, J. Barry, X. Yang, et al. The effect of anisotropic architecture on cell and tissue infiltration into tissue engineering scaffolds. Biomaterials, 27 (2006), pp. 5909-5917

[14] S.H. Oh, I.K. Park, J.M. Kim, J.H. Lee. In vitro and in vivo characteristics of PCL scaffolds with pore size gradient fabricated by a centrifugation method. Biomaterials, 28 (2007), pp. 1664-1671 
[15] G. Kim, J. Son, S. Park, W. Kim. Hybrid process for fabricating 3D hierarchical scaffolds combining rapid prototyping and electrospinning. Macromol. Rapid Commun., 29 (2008), pp. 1577-1581

[16] S. Park, G. Kim, Y.C. Jeon, Y. Koh, et al. 3D polycaprolactone scaffolds with controlled pore structure using a rapid prototyping system. J. Mater. Sci. Mater. Med., 20 (2009), pp. 229-234

[17] F. Zhao, P. Pathi, W. Grayson, Q. Xing, et al. Effects of oxygen transport on 3-d human mesenchymal stem cell metabolic activity in perfusion and static cultures: experiments and mathematical model. Biotechnol. Prog., 21 (2005), pp. 1269-1280

[18] B. Obradovic, J.H. Meldon, L.E. Freed, G. Vunjak-Novakovic. Glycosaminoglycan deposition in engineered cartilage: experiments and mathematical model. AIChE J., 46 (2000), pp. 18601871

[19] D. Nehring, P. Adamietz, N.M. Meenen, R. Pörtner. Perfusion cultures and modelling of oxygen uptake with three-dimensional chondrocyte pellets. Biotechnol. Tech., 13 (1999), pp. 701-706

[20] M. Pisu, N. Lai, A. Cincotti, F. Delogu, et al. A simulation model for the growth of engineered cartilage on polymeric scaffolds. Int. J. Chem. React. Eng., 1 (2003), p. 1084

[21] H. Kramers, G. Alberda. Frequency response analysis of continuous flow systems. Chem. Eng. Sci., 2 (1953), pp. 173-181

[22] C. Nicholson. Diffusion and related transport mechanisms in brain tissue. Rep. Prog. Phys., 64 (2001), p. 815 